Document downloaded from:

http://hdl.handle.net/10251/57515

This paper must be cited as:

Ayala Cabrera, D.; Herrera Fernández, AM.; Izquierdo Sebastián, J.; Pérez García, R. (2011). Location of buried plastic pipes by multi-agent support based on GPR images. Journal of Applied Geophysics. 75:679-686. doi:10.1016/j.jappgeo.2011.09.024.

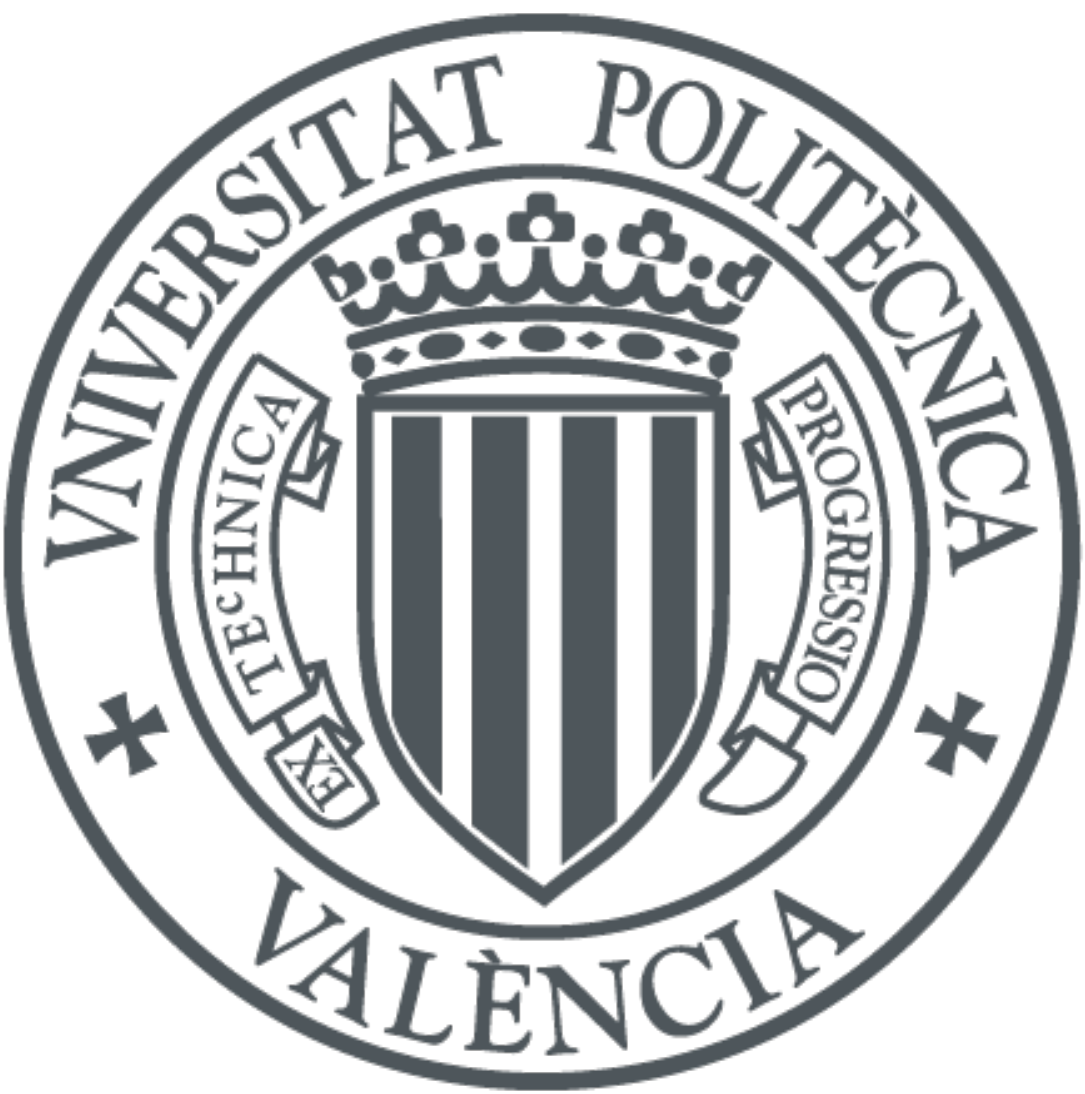

The final publication is available at

http://dx.doi.org/10.1016/j.jappgeo.2011.09.024

Copyright Elsevier

Additional Information 


\title{
Location of buried plastic pipes using multi-agent support based on GPR images ${ }^{\text {th }}$
}

\author{
David Ayala-Cabrera ${ }^{a}$, Manuel Herrera ${ }^{\mathrm{a}, *}$, Joaquín Izquierdo ${ }^{\mathrm{a}}$, Rafael \\ Pérez-García ${ }^{a}$ \\ ${ }^{a}$ FluIng-IMM, Universitat Politècnica de València, \\ C. de Vera s/n, 46022 Valencia, Spain
}

\begin{abstract}
This work focuses on the generation of tools to aid inspection and identify buried plastic pipes in water supply systems (WSS). In our study we use ground penetrating system (GPR) images as a non-destructive method of obtaining information without altering the system conditions and the environmental characteristics. A viability study for extracting features, and an approach to the above-mentioned application based on multi-agent systems are addressed in this paper. Firstly, we use intensive matrix manipulation of the GPR output for preprocessing the images. As a result, two matrices are produced that classify initial data based on the original radargram of the wave amplitude parameter. Then the plastic pipe characteristics that offer an enhanced likelihood of location are defined. This procedure is evaluated through two case-studies. One study corresponds to a simple case (one pipe) and the other corresponds to various pipes (made of different materials). Both cases were developed under controlled laboratory conditions. The obtained results are promising, and we show that automatic plastic pipe location has been achieved. The main contributions of the procedures proposed in this work are: firstly, highly skilled GPR prospection operators become unnecessary for plastic pipe location using GPR images; and secondly, we have opened a route to further classification that makes use of other methodologies.
\end{abstract}

\footnotetext{
This work has been supported by project IDAWAS, DPI2009-11591, of the Dirección General de Investigación of the Ministerio de Ciencia e Innovación of Spain, and ACOMP/2011/188 of the Conselleria de Educació of the Generalitat Valenciana.

*Corresponding author: mahefe@upv.es (Manuel Herrera)
} 
Keywords: Ground penetrating radar, pipe location, multi-agent systems, image processing.

\section{Introduction}

Pipes are one of the principal assets of a water supply system (WSS) and can be divided into transmission mains, distribution mains, and services. A variety of materials and technologies are used in the production of water supply pipes. The material of a particular pipe is closely related to the year of installation and the diameter. For large transmission pipelines (with diameters over $300 \mathrm{~mm}$ ), steel, mild steel cement-lined (MSCL), or pre-stressed concrete cylindrical pipes (PCCPs) are typically used. Older water distribution mains are usually made of cast iron or asbestos cement, while mainly ductile iron, polyethylene (PE), and polyvinyl chloride (PVC) are used for newer mains [31]. Plastic pipes (PE and PVC pipes) are frequently used in WSS, mainly in secondary networks (for service) and connections. Thus, a detailed knowledge of the characteristics of plastic pipes is necessary to achieve suitable control of a system. Many WSSs do not have information about the installed pipes or any possible changes made (some WSSs do not even have correct reference systems, or have just begun to implement reference systems). Taking into account network layout and pipe characteristics (such as diameter, material, and aging) enables making decisions regarding the technical management of WSS [4]. Managements may propose various targets such as: identification of illegal connections; planning of supply systems; simulation and network operation; adequate operation of plumbing systems; maintenance; rehabilitation and renewal of components; detection and leak control; applications of graphical information systems (GIS); and the evolution of pollutants in the networks.

There are several methods and techniques for inspecting and/or mapping pipes in WSSs, and these may be classified as destructive and non-destructive processes. Recent studies, such as several performed by the US Environmental Protection Agency (USEPA), underline the use of non-destructive tools as methodologies favouring technical management of WSS instead of other destructive testing methods [35]. The most commonly used non-destructive methods include: acoustic emission (AE); eddy currents (EC); electromagnetic methods (EM); impact-echo (IE); hammer sounding (HS); magnetic flux (MF); sonar methods (SM); ultrasonic testing (UT); pulsed induction 
methods (PIM); magnetic locators (ML); resistivity methods (RM); radio graphic testing (RT); pipeline inspection gauges (PIGs); and ground penetrating radar (GPR). The diversity of techniques shows that each has advantages and disadvantages. For example, EC and EM are used for inspecting metallic pipes, while AE and IE are used for concrete pipes [31]. PIM and RM are useful for locating metallic objects. Moreover, GPR has shown greater ability in detecting non-metallic pipes comparing with the above methods $[8,34]$, as well as inspecting and mapping other types of buried objects such as land mines [7] and archeologic vestiges [9], among others. However, even though information retrieval by GPR is worthwhile, the huge volume of generated information and the interpretation of data usually require high levels of skill and experience [35]. The principle behind GPR in detecting buried pipes exploits the differences between the dielectric properties of pipes and surrounding soil - and steel or cast iron pipes can be easily detected. However, if the pipe is made out of a material with dielectric properties close to that of soil, then detection may be difficult [23], as is the case with plastic pipes.

In this paper we work with GPR images, referencing ground profiles that can be used later (under a suitable metric) to inspect and map pipes. Several works have focused on the location of various metal components in GPR images $[9,11,15,26,33]$. As computer technology has improved, there have been increased efforts to develop tools based on intelligent systems. Thus, we find proposals for automatic pipe location in GPR images using neural networks $[1,12]$ and support vector machines (SVM) [29]. Pasolli et al. [28] proposed heuristic methods based on genetic algorithms. All of these works attempt to obtain clean images for locating objects through the implementation of automatic location methods and using an image cleaning process prior to the classification task $[22,27]$. However, there is an increasing use of plastic elements in WSSs and these interpretation methods need to be adapted to this current situation.

This work proposes a tool for identifying and locating plastic pipes in WSSs in GPR images. This methodology may be adapted for various pipe materials (metal, concrete, etc.) and is based on multi-agent systems working on a transformed ground penetrating radar image. The methodology was designed to be a reliable tool that does not require considerable experience and knowledge by users. The process is divided into three stages: a) transformation of images, b) multi-agent process, and c) location. The first stage transforms data obtained from GPR prospection into two types of im- 
ages termed by the authors: T14 and T15 matrices [2]. Secondly, we train the agents, by extracting plastic pipe characteristics, to automatically locate these pipes in the initial image.

The reminder of this paper is organised as follows. Section 2 presents a brief approach to GPR technology. This section also introduces a recently presented methodology to transform the wave signal space into a suitable framework for applying pipe location processes for GPR images. Section 3, presents a multi-agent process for an authomatic location of plastic pipes on GPR images. In Section 4 two instances of applications of a simple and a more complicated case-study are presented. Sections 5 and 6 close the paper describing some implementation issues and the main conclusions of the work, respectively.

\section{Transformation of GPR images}

There are multiple factors influencing GPR images. Each of the factors substantially alters the signal response to the passage though different materials. A comprehensive assessment of these factors produces a division into two groups: a) uncontrolled factors, and b) controlled factors. The first group includes environmental factors such as: topography; soil humidity; electromagnetic properties of the prospected medium (electrical conductivity, dielectric permittivity, and magnetic permeability); medium within which the electromagnetic wave propagates; depth of the pipes; etc. This group is not considered in our study for the surrounding medium of the buried pipes, since the test performed has been made under constant, uniform, and controlled laboratory conditions. The second group includes such factors as sampling conditions (GPR design, receiver performance, the employed antennas, etc.), data collection, pre-visualisation, storage and post-processing. This work is devoted to data post-processing, while other factors in this group remain constant.

This section focuses on post-processing data from traces and its subsequent transformation into images. The underlying reason for performing this task is to avoid noise sources generating new images when training intelligent systems (the multi-agent systems discussed in Section 3) to efficiently locate buried pipes. 


\subsection{An approach to GPR}

GPR is a non-invasive technique enabling buried objects to be detected without altering the environment [25]. Prospection is based on the emission of electromagnetic pulses from the surface which are propagated and reflected by any discontinuities encountered underground. This technique can reveal interfaces between the different materials, provided there is sufficient contrast between the dielectric properties of the materials. The energy of the reflected pulse is measured on the surface by receiving devices. The time needed for the pulse to travel from the surface and return provides a measurement of the depth of a reflector, given the positions of the source and receiver and the propagation speed of the pulses in the soil type [30]. Thus, a comparison between amplitudes of emitted and received pulses provides additional information about the composition of the subsoil [14]. These signals, called traces, are captured at fixed times and contain electromagnetic characteristics of the traversed medium. The subsequent accumulation of traces leads to what is known as radargrams, which are generally displayed using colour or grey scales to generate images.

GPR is a technique that has the advantage of high resolution and the ability to detect non-magnetic objects through environmental signals $[5,6]$. However, as with all GPR investigations there are limitations depending on the uncontrolled and controlled groups of factors, and other underground structures in urban areas. For example, soil penetration depends on soil type and antenna type. Soil moisture, as well as highly clay soils, will quickly attenuate the radar signal and decrease performance (i.e. dry sandy soils are best). High frequency antennas, in the order of 1 to $2 \mathrm{GHz}$, produce the best resolution (i.e., can find small objects), but can only achieve penetration of one to few meters. Low frequency antennas, in the order or 10 to $200 \mathrm{MHz}$, can achieve penetration up to tens of meters, depending on soil conditions, but may not be able to locate small objects or small diameter pipes [24, 21]. In addition, the use and interpretation of GPR data requires a high level of skill and experience [35, 10]. As a consequence, these costly problems have encouraged an increasing demand for the development of automated subsurface mapping techniques that are both accurate and rapid [29]. Our work is carried out with the objective of generating tools that do not require high levels of experience to acquire good results. In addition, we expect that this would be useful for the posterior implementation of an automatic system for inspecting and/or mapping the subsoil. Such tools would enable a better mapping of WSS components. 


\subsection{Introduction to the transformation methodology}

The transformation method used in the initial radargram (raw data) is based on the wave amplitude parameter. The raw (matrix) data is taken as input in the method and divided into ranges of values and rebuilt into new matrices. This method gathers various characteristics of the sought anomalies and produces clean images. The method builds the T14 and T15 matrices $[3,2]$ and comprises four phases: wave amplitude values (WAV) classification; WAV extraction; WAV accumulation; and WAV rebuilding. The matrices $T 14$ and $T 15$ constitute the output of the transformation and may be visualised as images (see Section 3.2). The four transformation phases are applied to each trace (column) of the radargram and are described next.

1. WAV classification. The captured wave amplitude values are separated into matrices resulting from some applied partition norm $(h=1000$, in the next case-study). As a result, we obtain the so-called $B^{(k)}$ matrices, where $k=1, \ldots, K+1, K$ being the maximum WAV in the raw data matrix.

2. WAV extraction. The $B^{(k)}$ matrices are the inputs of this phase, and the WAV are analysed taking into account their path. Thus, if the paths are towards higher WAVs, they are extracted and classified into so-called $D$ matrices. Otherwise, if the paths are toward lower WAVs, they are classified into so-called $U$ matrices.

The process continues after WAV extraction. There are two possible paths to be followed for each wave amplitude value, and these are established by the upper part $(\bar{D})$, and the lower part of the wave $(\underline{D})$, respectively. Similarly, we can obtain $\bar{U}$ and $\underline{U}$ regarding $U$ matrices. The WAV extraction from these matrices: $\bar{D}^{(c)}, \underline{D}^{(c)}, \bar{U}^{(c)}$ and, $\underline{U}^{(c)}$, represents the behavior assessment of each WAV profile. Where $C=$ $\{k: c+1 \leq k<K\}$ by $\bar{D}$ and $\underline{D}$, and $C=\{k: c+1 \leq k \leq K\}$ by $\bar{U}$ and $\underline{U}$.

3. WAV accumulation. Accumulating each of these $c$ matrices $\bar{D}$ by summing them, we obtain the T3 matrix. By similar processes, T5, T9 and $T 12$ are obtained from $\underline{D}, \bar{U}$ and $\underline{U}$ matrices, respectively.

4. Wave rebuilding. Finally, the sum of $T 3$ with $T 5$ and $T 9$ with $T 12$; gives the $T 14$ and $T 15$ matrices, respectively. The values in the matrices $T 14$ and $T 15$ are complementary. These matrices only match in the valleys and peaks. The values in these matrices represent the measurement of every WAV amplitude. 
An instance of the transformation methodology for one trace is shown in Figure 1.The figure shows, the $k$ columns, consecutively ascending, that compose the $B$ matrix and the $c$ columns for the $\bar{D}, \underline{D}, \bar{U}$ and $\underline{U}$ matrices.

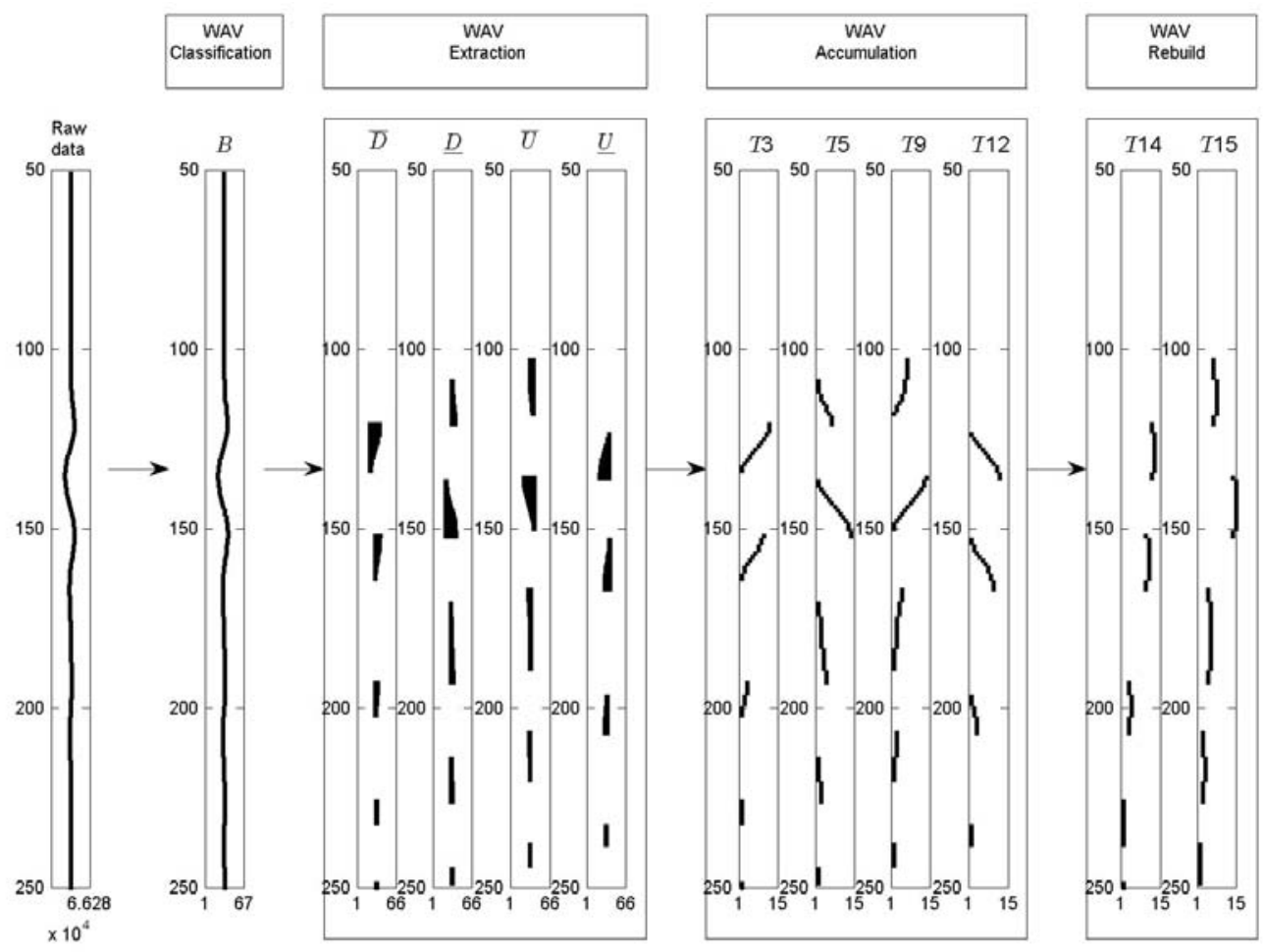

Figure 1: The transformation methodology on a trace.

\section{Automatic pipe location (multi-agent process)}

After data transformation, we apply multi-agent methodologies to select likely zones for locating PVC pipes in the modified GPR images. The proposed multi-agent process is composed of a training phase and an automatic location procedure (see Figure 2).

Firstly, we propose the type of agents to apply in this process. We then iteratively assess its behaviour, extracting the best location of buried plastic pipes. Finally, after applying this second stage, the process finishes with the pipe location in the image. These processes are detailed below in this section. 


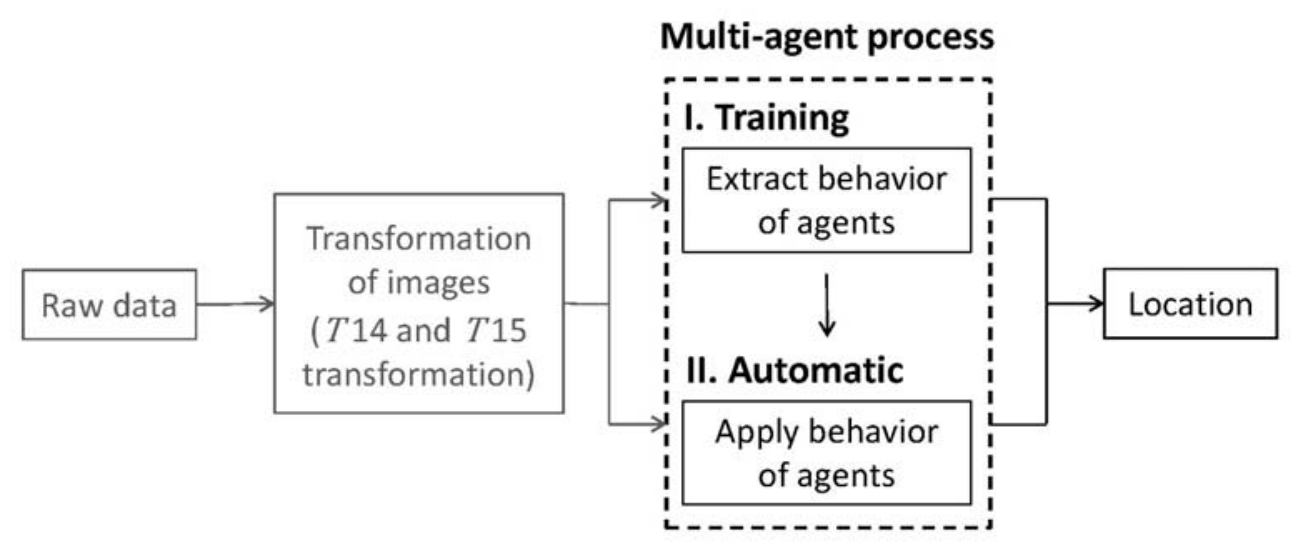

Figure 2: Automatic pipe location by multi-agent methods.

\subsection{A brief introduction to multi-agent systems}

A multi-agent system consists of a population of autonomous entities (agents) situated in a shared structured framework (environment) [36]. The system is based on such tools as game theory (e.g., setting the agent preferences by a utility function), economics, and biology, as well as artificial intelligence algorithms [32]. Other significant contributions of multi-agent systems to WSS may be found in the works of Gianetti et al. [13] who used agents to control the physical equipment of a water supply, or Izquierdo et al. [17, 18, 19], working on different aspects of WSS management, such as water-hammer control, or the establishment of criteria to divide the network into district metered areas.

In a system representing some reality (an image in our case) agents may be either exogenous or internal factors in the system. In the first case, agents are disseminated within the system to assess their immediate environments and make decisions about themselves, or their neighbouring agents, or their environment. In the second case, we understand that it is a system composed of subsystems at arbitrary nesting depths and achieving different levels of abstraction. Given a fixed level, the individual components will be the agents that decompose the whole system into different parts, and these are examined in a decentralised manner. Often, this is more efficient than working directly in some global approach.

Agents operate independently but they are also able to interact with their environment and coordinate with other agents. This coordination may imply 
cooperation if the agent society works towards common objectives. Thus, in a cooperative community, agents usually have individual capabilities which, when combined, will lead to solving the entire problem. But cooperation is not always possible and there are instances where agents are competitive and have divergent goals. In this later case, the agent should also take into account the actions of others. However, even if agents are able to act and achieve their goals by themselves, it may be beneficial to partially cooperate for a better performance, thereby forming coalitions. When coordinating activities, either in a cooperative or a competitive environment, negotiation may prove a suitable method to solve conflicts among agents. Negotiation may be seen as the process of identifying interactions based on communication and reasoning regarding the state and intentions of other agents [16].

Once agents have been defined and their relationships established, a schedule of combined actions defines processes occurring over time. These instructions are given to hundreds or thousands of agents that operate independently by interacting with their environment and coordinating among themselves. This coordination may imply cooperation (as in our case) if the agent society works towards common goals. Thus, in a cooperative community, agents usually have individual capabilities which, when combined, are able to efficiently solve a problem.

\subsection{Proposed multi-agent process}

The PVC pipe location algorithm is described in Figure 3. This figure shows that the process is based on images associated with the T14 (or T15) matrices and is composed of three phases: a) roll-on; b) termite-eating; and c) final detection.

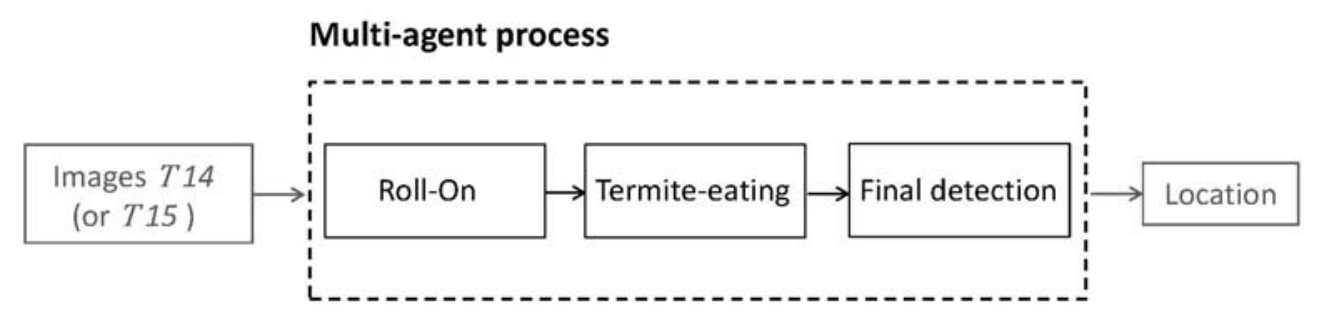

Figure 3: Phases of the proposed multi-agent process.

In the problem considered, the agents run on images $T 14$ (or T15). The underlying rationale behind this process consists in cleaning zones where the 
presence of a pipe is less likely, taking into account that the whole image is composed of blue and white pixels. Thus, we will discard areas where the values of the colour distribution mean that the presence of a pipe is unlikely. To establish this, let us consider the random variable $X$, which contains the value of a pixel colour and we will look for a probability such as:

$$
P\left(\left|\mu-\bar{X}_{\text {Obs }}\right|>\bar{X}_{\text {Color }}\right)<=t_{\text {ol }},
$$

where $\mu$ is the average colour in presence of a pipe, and $\bar{X}_{o b s}$ is the average colour observed in the area, and $t o l_{\alpha}$ is the error tolerance. If we fix $t_{0} l_{\alpha}$ we can obtain the critical values of $\bar{X}_{\text {Color }}$ by proposing the maximum and minimum values for deciding whether to erase, or not, each area under study. Zones are discarded by agents of different breeds that can cooperate with each other and their behaviour is based on Equation 1. The stages that compose this process are described below (and the implementation is detailed in Section 4):

1. Roll-on. A vertical array of agents covering the full height of the image is first defined. Each covers a squared area and measures the conditions to find a pipe. These agents move horizontally by scanning across the image width. After scanning a square, an agent deletes the corresponding sub-image if it corresponds to a zone where there is no pipe. The area is deleted if there are many blue (or white) pixels inside each inspected square.

2. Termite-eating. This first phase cannot distinguish where the pipes are located because the agents are constrained to fixed start positions and square dimensions. The creation of a new breed of agents will add flexibility to this method. We call these agents termites. They start randomly in a number of image pixels and their movements depend on the pixel colour where they are placed. Taking into account that pipes are blue coloured, the termites walk on blue pixels with care and go faster on white pixels. When the termite position is blue, an evaluation is carried out horizontally near the neighbouring pixels. This horizontal area will be deleted (eaten by the termite) if either too many or too few blue pixels are detected. This allows us to discriminate sets of blue pixels organised in shapes other than horizontal lines (such as the hyperbola shape in the image, which suggest the presence of a pipe).

3. The final detection phase is divided into various parts: firstly, we compute the borders of the undeleted figures. These borders are coloured 
with a colour of greater value than the corresponding blue in the RGB scale. Agents similar to those mentioned in phase 1 are then brought into action. However, these agents now have more flexibility in their movements, and cross along the image surface following random walks. This final process stops after an adequate number of iterations (which depends on the number of agents).

For the training stage, we propose a NetLogo interface [37] that gathers the three multi-agent processes in Section 5. This application is customisable using a simple menu (Figure 4). Users can interact with the process by changing the default parameters (e.g., number of iterations on each phase and the number of agents) and loading the file with their target image. The proposed interface is implemented in the NetLogo platform and the developed software is customisable by users. In this work, the interface was employed iteratively, determining suitable agent characteristics to detect and locate plastic pipes (PVC) in GPR images. Nevertheless, this interface may also be used to locate pipes made from other materials (including metal and concrete).

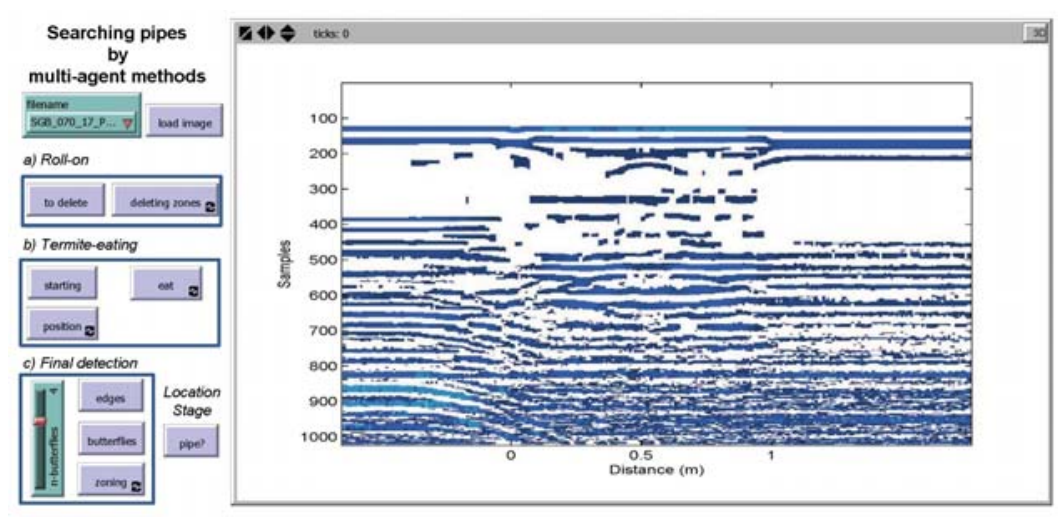

Figure 4: Proposed multi-agent interface.

Once a suitable agent-configuration is identified, we extract its behaviour to automatically detect pipes in other GPR images. Now, the remaining blue pixels (after the final detection phase) are coloured red, and a pipe is easily visualised in the plausible zone of its location when this image is overlapped with the $T 14$ (or T15) initial image. The complete automatic procedure is shown in Figure 5. 


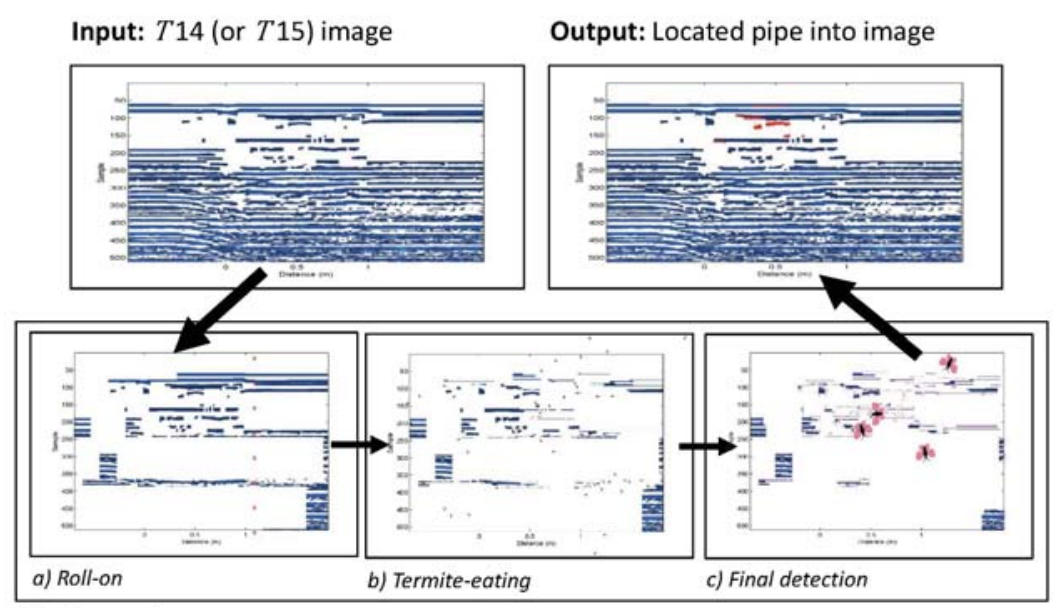

Multi-agent process

Figure 5: Evolution of the proposed multi-agent process.

\section{Experimental study}

The implementation viability of the multi-agent method proposed in Section 3.2 is evaluated in this section. To do this, we test two case-studies: a) a simple case; and b) a complex case. We used pipes commonly used in WSSs in the tests. The pipes were buried under controlled conditions in dry soil. The characteristics of the used pipes are shown in Table 1.

Table 1: Characteristics of the buried pipes used for testing.

\begin{tabular}{|l|c|c|c|}
\hline \multicolumn{1}{|c|}{ Material } & Index & Inner Diameter $(\mathrm{mm})$ & Outer Diameter $(\mathrm{mm})$ \\
\hline PVC & PVC & 100 & 110 \\
\hline Asbestos cement & Fib & 80 & 96 \\
\hline Cast Iron & Fund & 86 & 98 \\
\hline
\end{tabular}

The equipment used for these prospects corresponds to a monostatic antenna with a central frequency of $1.5 \mathrm{GHz}$. This indicates that it can be considered a good choice for urban underground exploration. Indeed, in this environment, the relevant structures (usually metallic or plastic pipes of many different sections) are relatively close to the ground surface [12]. Both cases are detailed below. 


\subsection{Customising the multi-agent location process}

To customise the automatic location process, the training stage proposes the following configuration for the multi-agent process:

1. In the first roll-on phase, the T14 image is covered with eight square agents that cross the image horizontally. While they are moving, the agents test the possibility of finding a pipe in their current position. These agents will delete the areas with low possibilities.

2. In the termite-eating phase, we generate 125 termites to check image positions in their random walks. These new agents will discard (eat) the horizontal blue stripes that are too large (more than 400 blue pixels) or too small (less than 50 blue pixels) to be part of a pipe image. To gain efficiency in their walks of 400 steps, the termites will move pixel to pixel if their current position is a blue pixel (pipes are formed by blue pixels), and jump ten pixels ahead if their position is white.

3. In the last final detection phase, we colour in purple (above blue in the RGB scale) the borders of the remaining images. We then start the process with four squared agents, as above, but moving randomly (200 iterations).

The customisation proposed is a consequence of the training stage where we evaluate twenty different agent configurations on $40 \mathrm{~T} 14$ and $40 \mathrm{~T} 15$ images of PVC pipes (20 for the simple case, see Subsection 4.2; and 20 for the more complex case, see Subsection 4.3). We select the best configuration (eight roll-on agents that cross the image, 125 termites - 400 iterations - and four square agents - 200 iterations). In more than $95 \%$ of the instances, the process successfully locates the PVC pipes for the simple case (this number diminishes in the complex case to $75 \%$ ). The false positive percentage is approximately $2 \%$ in the simple case and $10 \%$ in the more complex case. The final image obtained with this multi-agent process should be just the pipe (with maybe some noise around it). These pixels have been coloured in red and placed over the original image.

\subsection{Simple case}

This first case corresponds to a PVC pipe prospection (see Table 1). The proposed configuration for this case (Figure 6.a) was tested in a direction that was transversal to the pipe. The obtained raw data matrix is shown in 


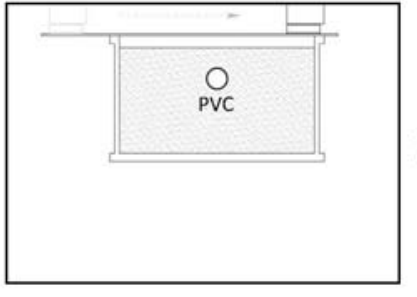

a).

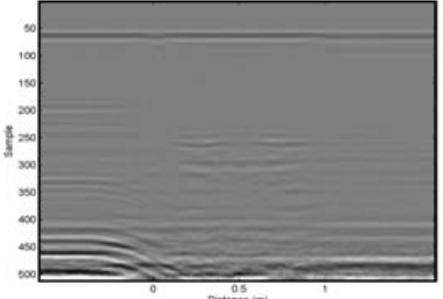

b).

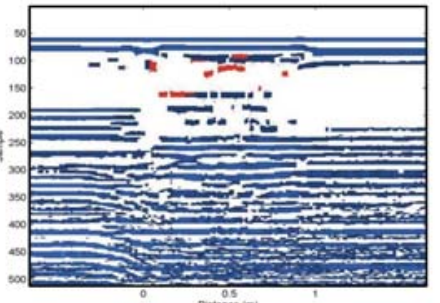

c).

Figure 6: Simple case. This shows the correspondence of the result obtained to the schema proposed.

greyscale (Figure 6.b). The results obtained, after applying the automatic location method proposed in this paper, are shown in Figure 6.c.

In the raw data image (Figure 6.b), we notice that the contrast of the borders of the PVC pipe are not strong enough to be seen immediately. This effect is a result of the low contrast between the permittivity of the soil and the buried pipe. This causes low colour intensity that prevents a clear PVC pipe demarcation in the image (being almost invisible). Figure 6.c shows the result after the application of the transformation methodology and the automatic location process. In the image we can also see the formation of a solid hyperbola (corresponding to the PVC pipe) that is only visible after the T14 (or T15) transformation. This hyperbola is successfully selected automatically.

\subsection{Complex case}

We prospected for three pipes in the more complex case. These pipes were buried simultaneously (at different elevations and positions). This second test attempts to obtain a better recreation of real-world street conditions, where pipes are often buried closely together. Figure 7.a shows the pipe distribution in the experimental tank and the raw image obtained by prospection (Figure 7.b). The methodology proposed in this paper was tested in this case. The results are shown in Figure 7.c.

Figure 7.b suggests traces of different pipes in response to the GPR signal. We can observe that the PVC pipe representation is similar to Figure 6.b with little or no demarcation boundaries. The cast iron pipe shows the better demarcation, while there are almost no traces of the asbestos cement pipe. 


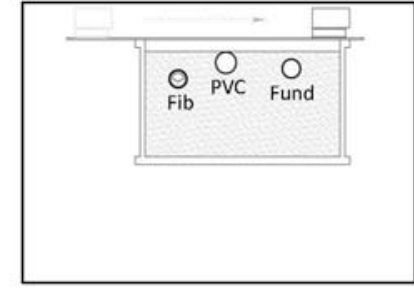

a).

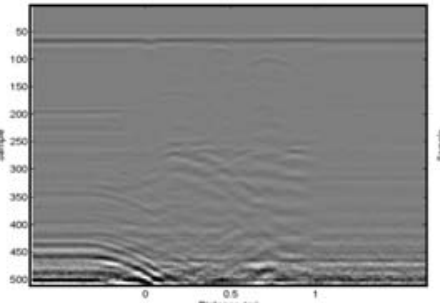

b).

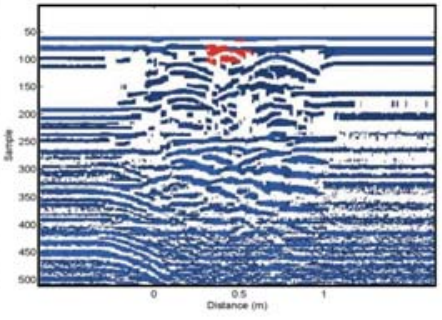

c).

Figure 7: Complex case. This shows the correspondence of the result obtained to the schema proposed.

After obtaining this data, we made a transformation using the proposed methodology. We then applied automatic plastic pipe location in the T14 image as shown in Figure 7.c (showing the hyperbolae of the three pipes). The closeness between the pipe means there is an overlap for the majority of the characteristics. This means it is more difficult to locate the pipes. Nevertheless, by applying the multi-agent process proposed above, we can discriminate the plastic pipe characteristics from the others.

\section{Implementation issues}

The algorithm have implemented on both Windows and Linux environments, using MatLab ${ }^{T M}$ to carry out the above treatment of matrices and the NetLogo platform as the multi-agent programming language. NetLogo offers a flexible and powerful tool for the programmer as it runs on the Java virtual machine and is similar to natural language in some ways (as we can see in Table 2, with an excerpt of the algorithm code for phase 2).

The captured GPR data is stored in *.dzt files (GPR survey format). The conversion of these files to Tab Delimited ASCII text format is performed using RTOAW freeware. With the information in a text file, the calculation, manipulation, and organisation are made in MatLab. The results of the process are images that are printed using the RGB colour scale, and processed with NetLogo, which, in turn, provides numerical and graphical output for export to other programs for further analysis. 
Table 2: Looking-for-pipes excerpt within stage 2 of the working algorithm.

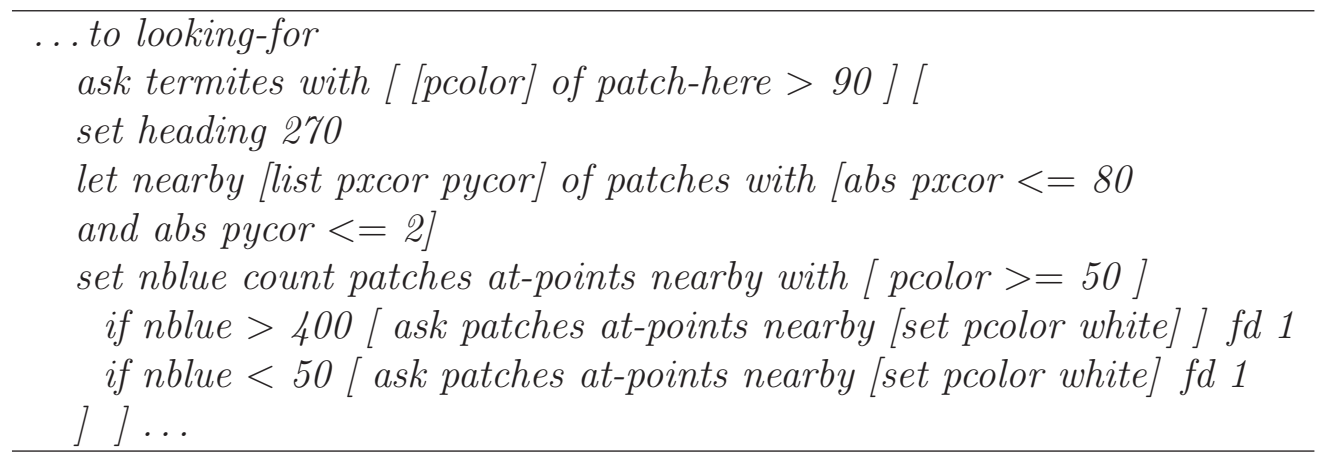

\section{Conclusions}

It is well-known that weakly reflective materials (PVC, PE) are difficult to identify without pre-processing the raw GPR captured images. Plastic pipes show weak values of wave amplitude after reflection, and reveal undefined contours along the boundaries. This paper introduces an automatic pipe location method based on multi-agent systems. This takes advantage of the quality offered by the $T 14$ and T15 image representations. The developed methodology is efficient with regard to the computational resources and accurate in its results (even in the challenging case of plastic pipes). The application is carried out by a customisable and simple software environment. It offers graphical and numerical results that could favour future exchanges between different software sources and help WSS managers gain a more accurate vision of their systems and so offer better service to users.

Finally, the proposed methodology to locate pipes in plastic pipe images was effective in both the case-studies. The obtained results show the viability of using multi-agent methods for locating (plastic) pipes in simple and complex cases. We should highlight that the proposed location process is a management tool that does not require user experience and can be used in both mapping and inspecting WSS pipe systems.

\section{References}

[1] Al-Nuaimy, W., Huang, Y., Nakhkash, M., Fang, M.T.C., Nguyen, V.T., Eriksen, A., 2000. Automatic detection of buried utilities and solid ob- 
jects with GPR using neural networks and pattern recognition, Journal of Applied Geophysics, vol. 43 (2-4), pp. 157-165.

[2] Ayala-Cabrera, D., Herrera, M., Montalvo, I., Pérez-García, R., 2011. Towards the visualization of water supply system components with GPR images, Journal of Mathematical and Computer Modelling, vol. 54 (7-8), pp. 1818-1822.

[3] Ayala-Cabrera, D., 2009. Caracterización de tuberías enterradas para redes de abastecimiento en servicio mediante el análisis de imágenes obtenidas con radar de subsuelo (Ground Penentrating Radar - GPR). Master Thesis. Polytechnic University of Valencia. Valencia (Spain).

[4] Bimpas, M., Amditis, A., Uzunoglu, N.K., 2010. Design and implementation of an integrated high resolution imaging ground penetrating radar for water pipe rehabilitation, Journal of Water Resources Management, Springer, pp. 1-12.

[5] Bonomo, N., De la Vega, M., 2010. El método de Georradar. URL http://www.gaia.df.uba.ar/Radar.pdf (Accessed on January 2010).

[6] Busquet, E., Casas, A., Pinto, V., Rivero, L., Sabadía, J.B., 2008. Geofísica Ambiental: Técnicas no destructivas para el reconocimiento de zonas contaminadas por vertidos, Acta Geológica Hispánica, vol. 30 (1-3), pp. 73-82.

[7] Chan, T.C.T., So, H.C., Ho, K.C., 2007. A study on two-sided linear prediction approach for land mine detection, Proc. of European Signal Processing Conference, Poznań (Poland), pp. 2414-2418.

[8] Cist, D.B., Schutz, A.E., 2001. State of the art for pipe \& leak detection DE-FC26-01NT41317 A low-cost GPR Gas Pipe \& leak detector. Geophysical survey systems, INC.

[9] Cukavac, M., Klemčić, G., Lazović, Č., 2008. Reconstruction of buried objects by implementation of ground penentrating radar technique: example on Roman Tomb in Brestovik (Serbia), Proc. of the International Conference on Geoarchaeology and Archaeomineralogy, pp. 333-338. 
[10] Dolittle, J.A., Bellantoni, N.F., 2010. The search for graves with groundpenetrating radar in Connecticut, Journal of Archaeological Science, pp. 941-949.

[11] Gader, P.D., Nelson, B.N., Frigui, H., Vaillette, G., Keller, J.M., 2000. Fuzzy logic detection of landmines with ground penetrating radar, Journal of Signal Processing, pp. 1069-1084.

[12] Gamba, P., Lossani, S., 2000. Neural Detection of pipe signatures in Ground Penetrating Radar Images. Journal IEEE Transactions on geoscience and remote sensing, vol. 38 (2) pp. 790-797.

[13] Gianetti, L., Maturana, F., Discenzo, F., 2005. Agent-based control of a municipal water system, Springer-Verlag.

[14] Göttshe, F.M., 1997. Identification of cavities by extraction of characteristic parameters from ground probing radar reflection data, $\mathrm{PhD}$. Thesis, Christian-Albrechts Universität, Zu Kiel, Germany.

[15] He, X.Q., Zhu, Z.Q., Liu, Q.Y., Lu, G.Y., 2009. Review of GPR rebar detection, Proc. of Progress In Electromagnetics Research Symposium (PIERS 2009), Beijing (China).

[16] Herrera, M., Izquierdo, J., Pérez-García, R., Ayala-Cabrera, D., 2010. Water supply clusters by multi-agent based approach, Proc. of Water Distribution System Analysis, Tucson (AZ, USA).

[17] Izquierdo, J., Herrera, M., Montalvo, I., Pérez-García, R., 2009. Agentbased division of water distribution systems into District Metered Areas, Proc. of International Conference on Software and Data Technologies, Sofia (Bulgaria).

[18] Izquierdo, J., Montalvo, I., Herrera, M., Pérez-García, R., 2010. Multiagent applications in Urban Hydraulics, Proc. of 16th European Conference on Mathematics for Industry, Wuppertal (Germany).

[19] Izquierdo, J., Montalvo, I., Pérez-García, R., Izquierdo, F.J., 2010. Hydraulic transient simulation using a multi-agent based approach, Proc. of Water Distribution System Analysis, Tucson (AZ, USA). 
[20] Jol, H.M., 2008. Ground penetrating radar: Theory and applications, First edition, Elsevier, Wisconsin University, USA.

[21] Lane, J.W., Buursink, M.L., Haeni, F.P., Versteeg, R.J., 2000. Evaluation of ground-penetrating radar to detect free-phase hydrocarbons in fractured rocks - results of numeerical modeling and physical experiments, Journal Ground Water, vol. 38 (6), pp. 929-938.

[22] Liu, C., Shen, L.C., 1991. Numerical simulation of subsurface radar for detecting buried pipes, IEEE Transactions on Geoscience and Remote Sensing, vol. 29 (5), pp. 795-798.

[23] Loulizi, A., 2001. Development of Ground penetrating radar signal modeling and implementation for transportation infrastructure assessment. PhD. Thesis, Virginia Polytechnic Institutee and State University, Blascksburg, Virginia.

[24] Malvar, L.J., Cline, G.D., 2004. Detecting Voids under Asphalt and Concrete Pavements. Transportation Systems, Fort Lauderdale, FL.

[25] Olhoeft, G.R., 2006. Ground Penetrating Radar. URL http://www.g-pr.com (Accessed on January 2010).

[26] Olhoeft, G.R., 2000. Maximizing the information return from Ground penetrating radar, Journal of Applied Geophysics, vol. 43 (2-4), pp. $175-187$.

[27] Pasolli, E., Melgani, F., Donelli, M., 2009. A pattern recognition system for extracting buried object characteristics in GPR images, Geoscience and Remote Sensing Symposium, Proc. of International Geoscience and Remote Sensing Symposium (IGARSS 2009), pp. 430-433.

[28] Pasolli, E., Melgani, F., Donelli, M., 2009. Automatic analysis of GPR images: A pattern-Recognition approach. Journal IEEE Transactions on Geoscience and Remote Sensing, vol. 47 (7), pp. 2206-2217.

[29] Pasolli, E., Melgani, F., Donelli, M., 2008. Automatic detection and classification of buried objects in GPR images using genetic algorithms and support vector machines, Proc. of International Geoscience and Remote Sensing Symposium (IGARSS 2008), pp. 525-528. 
[30] Pueyo, Ó., Zamora, S.A., Pocoví, J. A., 2005. Aplicación de la Prospección Geofísica por Georadar (GPR) a la Delimitación y Caracterización de Estructuras Arqueológicas. Ejemplo de las Ruinas del Convento Agustino de Fraga (Huesca), Geogaceta, vol. 38, pp. 131-134.

[31] Rizo, P., 2010. Water and wastewater pipe health monitoring: a review. Advances in Civil Engineering, Article ID 818597, 13 pages, doi:10.1155/2010/818597.

[32] Shoham, J. and Leyton-Brown, K., 2009. Multiagent Systems: Algorithmic, Game-Theoretic, and Logical Foundations, Cambridge University Press.

[33] Shaw, M.R., Millard, S.G., Molyneaux, T.C.K., Taylor, M.J., Bungey, J.H., 2005. Location of steel reinforcement in concrete using ground penetrating radar and neural networks, Proc. of International Conference on Structural Faults and Repairs (ROYAUME-UNI), vol. 38 (3), pp. 203-212.

[34] Takahashi, K., 2006. Detection and localization of subsurface objects by ground penetrating radar. PhD. Thesis, Tohoku University, Japan.

[35] Thomson, J., Wang, L., Royer, M., 2009. State of technology review report on condition assessment of ferrous water transmission and distribution systems, EPA United States Environmental Protection Agency, EPA/600/R-09/055.

[36] Weyns, D., Holvoet T., 2005. On the role of environments in multiagent systems, Informatica, vol. 29, pp. 405-421.

[37] Wilensky, U., 1999. NetLogo. http://ccl.northwestern.edu/netlogo/. Center for Connected Learning and Computer-Based Modeling, Northwestern University, Evanston, IL. 